\title{
Non-gaussianity from the bispectrum in general multiple field inflation
}

\author{
Frederico Arroja $\dagger^{*}$, Shuntaro Mizuno $\ddagger^{\dagger}$, and Kazuya Koyama $\dagger^{\ddagger}$ \\ $\dagger$ Institute of Cosmology and Gravitation, University of Portsmouth, Portsmouth PO1 2EG, UK \\ $\ddagger$ Research Center for the Early Universe (RESCEU), School of Science, \\ University of Tokyo, 7-3-1 Hongo, Bunkyo, Tokyo 113-0033, Japan
}

(Dated: June 15, 2008)

\begin{abstract}
We study the non-gaussianity from the bispectrum in multi-field inflation models with a general kinetic term. The models include the multi-field K-inflation and the multi-field Dirac-Born-Infeld (DBI) inflation as special cases. We find that, in general, the sound speeds for the adiabatic and entropy perturbations are different and they can be smaller than 1 . Then the non-gaussianity can be enhanced. The multi-field DBI-inflation is shown to be a special case where both sound speeds are the same due to a special form of the kinetic term. We derive the exact second and third order actions including metric perturbations. In the small sound speed limit and at leading order in the slow-roll expansion, we derive the three point function for the curvature perturbation which depends on both adiabatic and entropy perturbations. The contribution from the entropy perturbations has a different momentum dependence if the sound speed for the entropy perturbations is different from the adiabatic one, which provides a possibility to distinguish the multi-field models from single field models. On the other hand, in the multi-field DBI case, the contribution from the entropy perturbations has the same momentum dependence as the pure adiabatic contributions and it only changes the amplitude of the three point function. This could help to ease the constraints on the DBI-inflation models.
\end{abstract}

\section{INTRODUCTION}

The inflationary scenario succeeds to explain the origin of temperature fluctuations of the Cosmic Microwave Background $(\mathrm{CMB})$. The increasing precision of the measurements of the CMB enables us to distinguish between many inflationary models. The primordial fluctuations generated during inflation are nearly scale invariant and gaussian. Thus the deviation from the exact scale invariance and gaussianity will give valuable information in discriminating many possible models. Especially, non-gaussianity of the primordial fluctuations will provide powerful ways to constrain models (see e.g. 1] for a review). The simplest single field inflation models predict that the non-gaussianity of the fluctuations should be very difficult to be detected even in the future experiments such as Planck 2]. If we detect large non-gaussianity, this means that the simplest model of inflation would be rejected.

There are a few models where the primordial fluctuations generated during inflation have a large non-gaussianity. In the single field case, if the inflaton field has a non-trivial kinetic term, it is known that the non-gaussianity can be large. In $K$-inflation models where the kinetic term of the inflaton field is generic, the sound speed of the perturbations can be much smaller than 1 [3, 4]. This leads to a large non-gaussianity of the fluctuations. The Dirac-Born-Infeld (DBI) inflation, motivated by string theory, is another example [5, 6, 7, [8]. In DBI-inflation, the inflaton is identified with the position of a moving D3 brane whose dynamics is described by the DBI action. Again, due to the non-trivial form of the kinetic term, the sound speed can be smaller than 1 and the non-gaussianity becomes large [9, 10]. The third and fourth order actions for a single inflaton field with a generic kinetic term have been calculated by properly taking into account metric perturbations and three and four point functions have been calculated 10, 11, 12]. For the detailed observational consequences of single-field DBI-inflation see Refs. 13, 14, 15, 16, 17, 18, 19, 20].

Multi-field inflation models where the curvature perturbation is modified on large scales due to the entropy perturbations 21] have been also extensively studied recently. In the case of the standard kinetic term, it is not easy to generate large non-gaussianity from multi-field dynamics [22, 23] (see however Ref. 24]). In the DBI-inflation case, the position of the brane in each compact direction is described by a scalar field. Then DBI-inflation is naturally a multi-field model 25]. The effect of the entropy perturbations in the inflationary models based on string theory constructions in a slightly different context is also considered in [26, 27]. Recently, Huang et.al. calculated the bispectrum of the perturbations in multi-field DBI-inflation with the assumption that the kinetic term depends only on $X=-G^{I J} \partial_{\mu} \phi^{I} \partial^{\mu} \phi^{J} / 2$ where $\phi^{J}$ are the scalar fields $(I=1,2, \ldots)$ and $G_{I J}$ is the metric in the field space as in the case of K-inflation 28]. They found that in addition to the usual bispectrum of adiabatic perturbations, there exists

\footnotetext{
* Frederico.Arroja@port.ac.uk

$\dagger$ mizuno@resceu.s.u-tokyo.ac.jp

$\ddagger$ Kazuya.Koyama@port.ac.uk
} 
a new contribution coming from the entropy perturbations. Then they showed that the entropy field perturbations propagate with the speed of light and the contribution from the entropy perturbations is suppressed. This property can also be confirmed by the analysis of a more general class of multi-field models where the kinetic terms are given by arbitrary functions of X [29, 30]. However, Langlois et.al. pointed out that their assumption cannot be justified for the multi-field DBI-inflation [31]. Even though the action depends only on $X$ in the homogeneous background, there exit other kind of terms which contribute only to inhomogeneous perturbations. They find that this dramatically changes the behavior of the entropy perturbations. In fact, it was shown that the entropy perturbations propagate with the same sound speed as the adiabatic perturbations.

In this paper, we study a fairly general class of multi-field inflation models with a general kinetic term which includes K-inflation and DBI-inflation. We study the sound speeds of the adiabatic perturbations and entropy perturbations and clarify the difference between K-inflation and DBI-inflation. Then we calculate the third order action by properly taking into account the effect of gravity. Then three point functions at leading order in slow-roll and in the small sound speed limit are obtained. We can recover the results for K-inflation and DBI-inflation easily from this general result.

The structure of the paper is as follows. In section II, we describe our model and derive the equations in the background. In section III, we study the perturbations using the ADM formalism. The second and third order actions are derived by properly taking into account the metric perturbations. Then we decompose the perturbations into adiabatic and entropy directions and write down the action in terms of the decomposed fields. In section IV, we study the sound speed in several models including K-inflation and DBI-inflation. It is shown that in general, adiabatic and entropy sound speeds are different and both can be smaller than 1 . It is shown that the DBI action can be obtained by requiring that the sound speeds for the adiabatic and entropy perturbations are the same. In section $\mathrm{V}$, the third order action at leading order in slow-roll and in the small sound speed limit is obtained in terms of the decomposed fields. Then the three point functions are derived for a generalized model which includes K-inflation and DBI-inflation as particular cases. Section VI is devoted to the conclusion.

\section{THE MODEL}

We consider a very general class of models described by the following action

$$
S=\frac{1}{2} \int d^{4} x \sqrt{-g}\left[M_{P l}^{2} R+2 P\left(X^{I J}, \phi^{I}\right)\right],
$$

where $\phi^{I}$ are the scalar fields $(I=1,2, \ldots, N), M_{P l}$ is the Planck mass that we will set to unity hereafter, $R$ is the Ricci scalar and

$$
X^{I J} \equiv-\frac{1}{2} g^{\mu \nu} \partial_{\mu} \phi^{I} \partial_{\nu} \phi^{J}
$$

is the kinetic term, $g_{\mu \nu}$ is the metric tensor. We label the fields' Lagrangian by $P$ and we assume that it is a well behaved function. Greek indices run from 0 to 3 . Lower case Latin letters $(i, j, \ldots)$ denote spatial indices. Upper case Latin letters denote field indices.

The Einstein field equations in this model are

$$
G_{\mu \nu}=P g_{\mu \nu}+P_{, X I J} \partial_{\mu} \phi^{I} \partial_{\nu} \phi^{J} \equiv T_{\mu \nu}
$$

where $P_{, X^{I J}}$ denotes the derivative of $P$ with respect to $X^{I J}$ [32]. The generalized Klein-Gordon equation reads

$$
g^{\mu \nu}\left(P_{, X^{I J}} \partial_{\nu} \phi^{I}\right)_{; \mu}+P_{, J}=0
$$

where; denotes covariant derivative with respect to $g_{\mu \nu}$ and $P_{, J}$ denotes the derivative of $P$ with respect to $\phi^{J}$.

In the background, we are interested in flat, homogeneous and isotropic Friedmann-Robertson-Walker universes described by the line element

$$
d s^{2}=-d t^{2}+a^{2}(t) \delta_{i j} d x^{i} d x^{j},
$$

where $a(t)$ is the scale factor. The Friedmann equation and the continuity equation read

$$
3 H^{2}=E_{0},
$$




$$
\dot{E}_{0}=-3 H\left(E_{0}+P_{0}\right)
$$

where the Hubble rate is $H=\dot{a} / a, E_{0}$ is the total energy of the fields and it is given by

$$
E_{0}=2 X_{0}^{I J} P_{, X^{I J}}-P_{0}
$$

where a subscript zero denotes background quantities and $X_{0}^{I J}=1 / 2 \dot{\phi}_{0}^{I} \dot{\phi}_{0}^{J}$. The equations of motion for the scalar fields reduce to

$$
P_{, X^{I J}} \ddot{\phi}^{I}+\left(3 H P_{, X^{I J}}+\dot{P}_{, X^{I J}}\right) \dot{\phi}^{I}-P_{, J}=0 .
$$

\section{PERTURBATIONS}

In this section, we will consider perturbations of the background (5) beyond linear order. For this purpose, we will construct the action at second and third order in the perturbations and it is convenient to use the ADM metric formalism [10, 29, 30, 33, 34, 35, 36]. The ADM line element reads

$$
d s^{2}=-N^{2} d t^{2}+h_{i j}\left(d x^{i}+N^{i} d t\right)\left(d x^{j}+N^{j} d t\right)
$$

where $N$ is the lapse function, $N^{i}$ is the shift vector and $h_{i j}$ is the $3 \mathrm{D}$ metric. The action (11) becomes

$$
S=\frac{1}{2} \int d t d^{3} x \sqrt{h} N\left({ }^{(3)} R+2 P\left(X^{I J}, \phi^{I}\right)\right)+\frac{1}{2} \int d t d^{3} x \sqrt{h} N^{-1}\left(E_{i j} E^{i j}-E^{2}\right) .
$$

The tensor $E_{i j}$ is defined as

$$
E_{i j}=\frac{1}{2}\left(\dot{h}_{i j}-\nabla_{i} N_{j}-\nabla_{j} N_{i}\right)
$$

and it is related to the extrinsic curvature by $K_{i j}=N^{-1} E_{i j} . \nabla_{i}$ is the covariant derivative with respect to $h_{i j} \cdot X^{I J}$ can be written as

$$
X^{I J}=-\frac{1}{2} h^{i j} \partial_{i} \phi^{I} \partial_{j} \phi^{J}+\frac{N^{-2}}{2} v^{I} v^{J}
$$

where $v^{I}$ is defined as

$$
v^{I} \equiv \dot{\phi}^{I}-N^{j} \nabla_{j} \phi^{I}
$$

The Hamiltonian and momentum constraints are respectively

$$
\begin{aligned}
{ }^{(3)} R+2 P-2 N^{-2} P_{, X^{I J}} v^{I} v^{J}-N^{-2}\left(E_{i j} E^{i j}-E^{2}\right) & =0, \\
\nabla_{j}\left(N^{-1} E_{i}^{j}\right)-\nabla_{i}\left(N^{-1} E\right) & =N^{-1} P_{, X^{I J}} v^{I} \nabla_{i} \phi^{J} .
\end{aligned}
$$

We decompose the shift vector $N^{i}$ into scalar and intrinsic vector parts as

$$
N_{i}=\tilde{N}_{i}+\partial_{i} \psi
$$

where $\partial_{i} \tilde{N}^{i}=0$, here and in the rest of the section indices are raised with $\delta_{i j}$.

\section{A. Perturbations in the uniform curvature gauge}

In the uniform curvature gauge, the $3 \mathrm{D}$ metric takes the form

$$
\begin{aligned}
& h_{i j}=a^{2} \delta_{i j}, \\
& \phi^{I}(x, t)=\phi_{0}^{I}(t)+Q^{I}(x, t),
\end{aligned}
$$

where $Q^{I}$ denotes the field perturbations. In the following, we will usually drop the subscript " 0 " on $\phi_{0}^{I}$ and simply identify $\phi^{I}$ as the homogeneous background fields unless otherwise stated. 
We expand $N$ and $N^{i}$ in powers of the perturbation $Q^{I}$

$$
\begin{aligned}
N & =1+\alpha_{1}+\alpha_{2}+\cdots, \\
\tilde{N}_{i} & =\tilde{N}_{i}^{(1)}+\tilde{N}_{i}^{(2)}+\cdots, \\
\psi & =\psi_{1}+\psi_{2}+\cdots
\end{aligned}
$$

where $\alpha_{n}, \tilde{N}_{i}^{(n)}$ and $\psi_{n}$ are of order $\left(Q^{I}\right)^{n}$. At first order in $Q^{I}$, a particular solution for equations (15) is:

$$
\begin{aligned}
\alpha_{1} & =\frac{P_{, X^{I J}}}{2 H} \dot{\phi}^{I} Q^{J}, \quad \tilde{N}_{i}^{(1)}=0, \\
\partial^{2} \psi_{1} & =\frac{a^{2}}{2 H}\left[-6 H^{2} \alpha_{1}+P_{, K} Q^{K}-\dot{\phi}^{I} \dot{\phi}^{J} P_{, X^{I J} K} Q^{K}+\left(P_{, X^{I J}}+\dot{\phi}^{L} \dot{\phi}^{M} P_{, X^{L M} X^{I J}}\right)\left(\dot{\phi}^{I} \dot{\phi}^{J} \alpha_{1}-\dot{\phi}^{J} \dot{Q}^{I}\right)\right] .
\end{aligned}
$$

The second order action is calculated as

$$
\begin{aligned}
S_{(2)}=\int d t d^{3} x \frac{a^{3}}{2}[ & X_{1}^{I J} X_{1}^{L M} P_{, X^{I J} X^{L M}}+P_{, X^{I J}} \dot{Q}^{I} \dot{Q}^{J}-a^{-2} P_{, X^{I J}} \partial^{i} Q^{I} \partial_{i} Q^{J}-\frac{3}{2} \dot{\phi}^{J} \dot{\phi}^{L} P_{, X^{I J}} P_{, X^{L M}} Q^{I} Q^{M} \\
& +\frac{\dot{\phi}^{J} P_{, X^{I J}} Q^{I}}{H}\left(P_{, X^{L M}} X_{1}^{L M}+P_{, K} Q^{K}\right)+2 P_{, X^{I J} K} Q^{K}\left(-\dot{\phi}^{I} \dot{\phi}^{J} \alpha_{1}+\dot{\phi}^{I} \dot{Q}^{J}\right)+P_{, K L} Q^{K} Q^{L} \\
& \left.+P_{, X^{I J}}\left(3 \dot{\phi}^{I} \dot{\phi}^{J} \alpha_{1}^{2}-4 \alpha_{1} \dot{\phi}^{I} \dot{Q}^{J}\right)\right]
\end{aligned}
$$

where

$$
X_{1}^{I J} \equiv-\alpha_{1} \dot{\phi}^{I} \dot{\phi}^{J}+\dot{\phi}^{(I} \dot{Q}^{J)}
$$

After integrating by parts in the action and employing the background field equations, the second order action can be finally written in the rather simple form

$$
\begin{aligned}
S_{(2)}= & \frac{1}{2} \int d t d^{3} x a^{3}\left[\left(P_{, X^{I J}}+P_{, X^{I K} X^{J L}} \dot{\phi}^{K} \dot{\phi}^{L}\right) \dot{Q}^{I} \dot{Q}^{J}\right. \\
& \left.-\frac{1}{a^{2}} P_{, X^{I J}} \partial_{i} Q^{I} \partial^{i} Q^{J}-\mathcal{M}_{I J} Q^{I} Q^{J}+\mathcal{N}_{I J} Q^{J} \dot{Q}^{I}\right],
\end{aligned}
$$

with the effective squared mass matrix

$$
\begin{aligned}
\mathcal{M}_{I J}= & -P_{, I J}+\frac{X^{L M}}{H} \dot{\phi}^{K}\left(P_{, X^{J K}} P_{, I X^{L M}}+P_{, X^{I K}} P_{, J X^{L M}}\right) \\
& -\frac{1}{H^{2}} X^{M N} X^{P Q} P_{, X^{M N} X^{P Q}} P_{, X^{I K}} P_{, X^{J L} \dot{\phi}^{K}} \dot{\phi}^{L} \\
& -\frac{1}{a^{3}} \frac{d}{d t}\left[\frac{a^{3}}{H} P_{, X^{I K}} P_{, X^{J L}} \dot{\phi}^{K} \dot{\phi}^{L}\right], \\
\mathcal{N}_{I J}= & 2\left(P_{, J X^{I K}}-\frac{X^{M N}}{H} P_{, X^{I K} X^{M N}} P_{, X^{J L}} \dot{\phi}^{L}\right) \dot{\phi}^{K} .
\end{aligned}
$$

In the same way, the third order action is given by

$$
\begin{aligned}
S_{(3)}= & \int d t d^{3} x a^{3}\left[\left[3 H^{2} \alpha_{1}^{2}+\frac{2 H}{a^{2}} \alpha_{1} \partial^{2} \psi_{1}+\frac{1}{2 a^{4}}\left(\partial^{2} \psi_{1} \partial^{2} \psi_{1}-\partial_{i} \partial_{j} \psi_{1} \partial^{i} \partial^{j} \psi_{1}\right)\right] \alpha_{1}\right. \\
& +\left[-\frac{1}{2} \alpha_{1}^{3} \dot{\phi}^{I} \dot{\phi}^{J}+\alpha_{1}^{2} \dot{\phi}^{I} \dot{Q}^{J}+a^{-2} \alpha_{1} \dot{\phi}^{I} \partial^{i} \psi_{1} \partial_{i} Q^{J}-\frac{\alpha_{1}}{2} \dot{Q}^{I} \dot{Q}^{J}-a^{-2} \partial_{i} Q^{J}\left(\dot{Q}^{I} \partial^{i} \psi_{1}+\frac{1}{2} \alpha_{1} \partial^{i} Q^{I}\right)\right] P_{, X^{I J}} \\
& +\left[\alpha_{1}^{2} \dot{\phi}^{I} \dot{\phi}^{J}-\frac{3}{2} \alpha_{1} \dot{\phi}^{I} \dot{Q}^{J}+\frac{1}{2} \dot{Q}^{I} \dot{Q}^{J}-a^{-2} \partial_{i} Q^{J}\left(\dot{\phi}^{I} \partial^{i} \psi_{1}+\frac{1}{2} \partial^{i} Q^{I}\right)\right] X_{1}^{L M} P_{, X^{I J} X^{L M}} \\
& +\left[\frac{1}{2} \alpha_{1}^{2} \dot{\phi}^{I} \dot{\phi}^{J}-\alpha_{1} \dot{\phi}^{I} \dot{Q}^{J}+\frac{1}{2} \dot{Q}^{I} \dot{Q}^{J}-a^{-2} \partial_{i} Q^{J}\left(\dot{\phi}^{I} \partial^{i} \psi_{1}+\frac{1}{2} \partial^{i} Q^{I}\right)\right] P_{, X^{I J}{ }_{K} Q^{K}} \\
& +\frac{\alpha_{1}}{2} P_{, I J} Q^{I} Q^{J}+\frac{1}{6} X_{1}^{I J} X_{1}^{L M} X_{1}^{Q R} P_{, X^{I J} X^{L M} X^{Q R}}+\frac{1}{2} X_{1}^{I J} X_{1}^{L M} P_{, X^{I J} X^{L M} K} Q^{K} \\
& \left.+\frac{1}{2} X_{1}^{I J} P_{, X^{I J} L M} Q^{L} Q^{M}+\frac{1}{6} P_{, I J K} Q^{I} Q^{J} Q^{K}\right]
\end{aligned}
$$




\section{B. Decomposition into adiabatic and entropy perturbations}

We can decompose the perturbations into the instantaneous adiabatic and entropy perturbations, where the adiabatic direction corresponds to the direction of the background fields' evolution while the entropy directions are orthogonal to this [37]. For this purpose, following [29], we introduce an orthogonal basis $e_{n}^{I}(n=1,2, \ldots, N)$ in the field space. The orthonormal condition is defined as

$$
P_{, X^{I J}} e_{n}^{I} e_{m}^{J}=\delta_{n m}
$$

so that the gradient term $P_{, X^{I J}} \partial_{i} Q^{I} \partial^{i} Q^{J}$ is diagonalized [38]. We pick up the adiabatic vector as

$$
e_{1}^{I}=\frac{\dot{\phi}^{I}}{\sqrt{P_{, X^{J K}} \dot{\phi}^{J} \dot{\phi}^{K}}},
$$

which satisfies the normalization given by Eq. (28). The field perturbations are decomposed on this basis as

$$
Q^{I}=Q_{n} e_{n}^{I}
$$

We defined the matrix $Z_{m n}$ which describes the time variation of the basis as

$$
\dot{e}_{n}^{I}=e_{m}^{I} Z_{m n}
$$

which satisfies $Z_{m n}=-Z_{n m}-\dot{P}_{X^{I J}} e_{m}^{I} e_{n}^{J}$ as a consequence of $\left(P_{, X^{I J}} e_{n}^{I} e_{m}^{J}\right)^{\cdot}=0$.

In terms of the decomposed fields, the second order action (24) can be rewritten as

$$
\begin{aligned}
S_{(2)}= & \frac{1}{2} \int d t d^{3} x a^{3}\left[\mathcal{K}_{m n}\left(D_{t} Q_{m}\right)\left(D_{t} Q_{n}\right)-\frac{1}{a^{2}} \delta_{m n} \partial_{i} Q_{m} \partial^{i} Q_{n}\right. \\
& \left.-\mathcal{M}_{m n} Q_{m} Q_{n}+\mathcal{N}_{m n} Q_{n}\left(D_{t} Q_{m}\right)\right]
\end{aligned}
$$

where

$$
\begin{aligned}
D_{t} Q_{m} & \equiv \dot{Q}_{m}+Z_{m n} Q_{n} \\
\mathcal{K}_{m n} & \equiv \delta_{m n}+\left(P_{, X^{M N}} \dot{\phi}^{M} \dot{\phi}^{N}\right) P_{, X^{I K} X^{J L}} e_{1}^{I} e_{n}^{K} e_{1}^{J} e_{m}^{L} \\
\mathcal{M}_{m n} & \equiv \mathcal{M}_{I J} e_{m}^{I} e_{n}^{J} \\
\mathcal{N}_{m n} & \equiv \mathcal{N}_{I J} e_{m}^{I} e_{n}^{J} .
\end{aligned}
$$

From the constructions, $\mathcal{K}_{m n}, \mathcal{M}_{m n}$ and $\mathcal{N}_{m n}$ are symmetric with respect to $m$ and $n$. The explicit form of the effective squared mass matrix in this representation is

$$
\begin{aligned}
\mathcal{M}_{m n}= & -P_{, m n}+\frac{1}{H}\left(P_{, X^{K L}} \dot{\phi}^{K} \dot{\phi}^{L}\right)^{3 / 2}\left(P_{, m X^{M N}} e_{1}^{M} e_{1}^{N}\right) \delta_{n 1} \\
& -\frac{1}{4 H^{2}}\left(P_{, X^{K L}} \dot{\phi}^{K} \dot{\phi}^{L}\right)^{3}\left(P_{, X^{M N} X^{P Q}} e_{1}^{M} e_{1}^{N} e_{1}^{P} e_{1}^{Q}\right) \delta_{m 1} \delta_{n 1} \\
& -\frac{1}{a^{3}} \frac{d}{d t}\left[\frac{a^{3}}{H}\left(P_{, X^{M N}} \dot{\phi}^{M} \dot{\phi}^{N}\right) P_{, X^{I K}} P_{, X^{J L}} e_{1}^{K} e_{1}^{L}\right] e_{m}^{I} e_{n}^{J} \\
\mathcal{N}_{m n} \equiv & -\frac{1}{H}\left(P_{, X^{P Q}} \dot{\phi}^{P} \dot{\phi}^{Q}\right)^{2}\left(P_{, X^{K L} X^{M N}} e_{m}^{K} e_{1}^{L} e_{1}^{M} e_{1}^{N}\right) \delta_{n 1} \\
& +2 \sqrt{P_{, X^{L M}} \dot{\phi}^{L} \dot{\phi}^{M}}\left(P_{, n X^{I K}} e_{m}^{I} e_{1}^{K}\right)
\end{aligned}
$$

where $P_{, m n} \equiv P_{, I J} e_{m}^{I} e_{n}^{J}$ and $P_{, n X^{I K}} \equiv P_{, J X^{I K}} e_{n}^{J}$.

The equation of motion is obtained as

$$
\begin{aligned}
& \frac{1}{a^{3}} \frac{d}{d t}\left[a^{3}\left(2 \mathcal{K}_{m r} D_{t} Q_{m}+\mathcal{N}_{m r} Q_{m}\right)\right]-\left(2 \mathcal{K}_{m n} Z_{n r}+\mathcal{N}_{m r}\right) D_{t} Q_{m} \\
& -\left(2 \mathcal{M}_{m r}+\mathcal{N}_{m n} Z_{m r}\right) Q_{m}+\frac{2}{a^{2}} \partial^{2} Q_{r}=0
\end{aligned}
$$




\section{LINEAR PERTURBATIONS}

In this section, we study the linear order perturbations using the second order action derived in the previous section.

\section{A. K-inflation}

Let us consider K-inflation models where $P\left(X^{I J}, \phi^{I}\right)$ is a function of only the trace $X=X^{I J} G_{I J}\left(\phi^{K}\right)$ of the kinetic terms where $G_{I J}\left(\phi^{K}\right)$ is a metric in the field space:

$$
P\left(X^{I J}, \phi^{I}\right)=\tilde{P}\left(X, \phi^{I}\right)
$$

The derivatives of $\mathrm{P}$ can be evaluated as

$$
\begin{aligned}
P_{, X^{I J}}= & G_{I J} \tilde{P}_{, X}, \\
P_{, I}= & \frac{1}{2} G_{J K, I} \dot{\phi}^{J} \dot{\phi}^{K} \tilde{P}_{, X}+\tilde{P}_{, I}, \\
P_{, X^{I J} X^{K L}}= & G_{I J} G_{K L} \tilde{P}_{, X X}, \\
P_{, X^{I J} K}= & \frac{1}{2} G_{L M, K} \dot{\phi}^{L} \dot{\phi}^{M} G_{I J} \tilde{P}_{, X X}+G_{I J, K} \tilde{P}_{, X}+G_{I J} \tilde{P}_{, X K}, \\
P_{, I J}= & \frac{1}{4} G_{K L, I} G_{M N, J} \dot{\phi}^{K} \dot{\phi}^{L} \dot{\phi}^{M} \dot{\phi}^{N} \tilde{P}_{, X X}+\frac{1}{2} G_{K L, I J} \dot{\phi}^{K} \dot{\phi}^{L} \tilde{P}_{, X} \\
& +\frac{1}{2} \dot{\phi}^{M} \dot{\phi}^{N}\left(G_{M N, J} \tilde{P}_{, X I}+G_{M N, I} \tilde{P}_{, X J}\right)+\tilde{P}_{, I J},
\end{aligned}
$$

and the sound speed is defined as

$$
c_{s}^{2} \equiv \frac{\tilde{P}_{, X}}{\tilde{P}_{, X}+2 X \tilde{P}_{, X X}} .
$$

In terms of the decomposed field, the second order action can be written as

$$
\begin{aligned}
S_{(2)}= & \frac{1}{2} \int d t d^{3} x a^{3}\left[\left\{\delta_{m n}+\left(\frac{1}{c_{s}^{2}}-1\right) \delta_{1 m} \delta_{1 n}\right\}\left(D_{t} Q_{m}\right)\left(D_{t} Q_{n}\right)\right. \\
& \left.-\frac{1}{a^{2}} \delta_{m n} \partial_{i} Q_{m} \partial^{i} Q_{n}-\mathcal{M}_{m n} Q_{m} Q_{n}+\mathcal{N}_{m n} Q_{n}\left(D_{t} Q_{m}\right)\right]
\end{aligned}
$$

where we do not show the explicit forms of $\mathcal{M}_{m n}$ and $\mathcal{N}_{m n}$.

The sound speed agrees with the adiabatic sound speed defined by $c_{s}^{2}=d P / d E$. The fact that the sound speeds for the entropy perturbations are unity has been recognized in Ref. [29]. This is because the non-trivial second derivative of $P$ only affects the adiabatic perturbations, which is the consequence of the fact that the entropy field $s$ satisfies $\dot{s}=0$ in the background and thus it has no first order perturbations.

\section{B. DBI-inflation}

An interesting class of models is the DBI-inflation which describes the motion of a D3 brane in a higher dimensional spacetime. The DBI action is given by

$$
S=-\int d^{4} x \frac{1}{f\left(\phi^{K}\right)} \sqrt{-\operatorname{det}\left[g_{\mu \nu}+f\left(\phi^{K}\right) G_{I J}\left(\phi^{K}\right) \partial_{\mu} \phi^{I} \partial_{\nu} \phi^{J}\right]} .
$$

Recently it was pointed out by Ref. [31] that the multi-field DBI-inflation is not included in the multi-field K-inflation discussed in the previous subsection. Indeed, $P\left(X^{I J}\right)$ is not a function of $X$, but it is given by

$$
P\left(X^{I J}, \phi^{I}\right)=\tilde{P}\left(\tilde{X}, \phi^{I}\right), \quad \tilde{X}=\frac{(1-\mathcal{D})}{2 f},
$$


where

$$
\begin{aligned}
\mathcal{D} & =\operatorname{det}\left(\delta_{J}^{I}-2 f X_{J}^{I}\right) \\
& =1-2 f G_{I J} X^{I J}+4 f^{2} X_{I}^{[I} X_{J}^{J]}-8 f^{3} X_{I}^{[I} X_{J}^{J} X_{K}^{K]}+16 f^{4} X_{I}^{[I} X_{J}^{J} X_{K}^{K} X_{L}^{L]} .
\end{aligned}
$$

In the background, $\tilde{X}=\underset{\tilde{P}}{X}$. However, this does not mean that the full action is a function of $X$ only. The DBI action takes a specific form of $\tilde{P}$

$$
\tilde{P}\left(\tilde{X}, \phi^{I}\right)=-\frac{1}{f}(\sqrt{1-2 f \tilde{X}}-1)-V\left(\phi^{I}\right),
$$

where we allow for a potential $V\left(\phi^{I}\right)$. The sound speed is defined as

$$
c_{s}^{2} \equiv \frac{\tilde{P}_{, \tilde{X}}}{\tilde{P}_{, \tilde{X}}+2 X \tilde{P}_{, \tilde{X} \tilde{X}}} .
$$

The derivatives of $P$ can be calculated as

$$
\begin{aligned}
P_{, X^{I J}} & =\tilde{P}_{, \tilde{X}}\left(\frac{d \tilde{X}}{d X^{I J}}\right) \\
P_{, X^{I J} X^{K L}} & =\tilde{P}_{, \tilde{X} \tilde{X}}\left(\frac{d \tilde{X}}{d X^{I J}}\right)\left(\frac{d \tilde{X}}{d X^{K L}}\right)+\tilde{P}_{, \tilde{X}}\left(\frac{d^{2} \tilde{X}}{d X^{I J} d X^{K L}}\right),
\end{aligned}
$$

where

$$
\begin{aligned}
\frac{d \tilde{X}}{d X^{I J}} & =c_{s}^{2} G_{I J}+2 f X_{I J} \\
\frac{d^{2} \tilde{X}}{d X^{I J} d X^{K L}} & =-2 f\left(G_{I J} G_{K L}-\frac{1}{2} G_{I K} G_{J L}-\frac{1}{2} G_{I L} G_{J K}\right)+O\left(X^{I J}\right) .
\end{aligned}
$$

Here we do not explicitly write down the higher order terms in $X^{I J}$ in the second derivative as they will not contribute to the final result. In the following, we will omit these terms. We can also show that

$$
\begin{aligned}
P_{, I}= & \frac{1}{2} G_{J K, I} \dot{\phi}^{J} \dot{\phi}^{K} \tilde{P}_{, \tilde{X}}+\tilde{P}_{, I}, \\
P_{, I J}= & \frac{1}{4} G_{K L, I} G_{M N, J} \dot{\phi}^{K} \dot{\phi}^{L} \dot{\phi}^{M} \dot{\phi}^{N} \tilde{P}_{, \tilde{X} \tilde{X}}+\frac{1}{2} G_{K L, I J} \dot{\phi}^{K} \dot{\phi}^{L} \tilde{P}_{, \tilde{X}} \\
& +\frac{1}{2} \dot{\phi}^{M} \dot{\phi}^{N}\left(G_{M N, J} \tilde{P}_{, \tilde{X}_{I}}+G_{M N, I} \tilde{P}_{, \tilde{X} J}\right)+\tilde{P}_{, I J}, \\
P_{, X^{I J} K}= & \left((1-2 f X) G_{I J, K}-2 f_{, K} X G_{I J}-2 f G_{L M, K} X^{L M} G_{I J}\right. \\
& \left.2 f_{, K} X_{I J}+2 f G_{I L, K} X_{J}^{L}+2 f G_{J M, K} X_{I}^{M}\right) \tilde{P}_{, \tilde{X}} \\
& +\left(c_{s}^{2} G_{I J}+2 f X_{I J}\right)\left(\frac{1}{2} G_{L M, K} \dot{\phi}^{L} \dot{\phi}^{M} \tilde{P}_{, \tilde{X} \tilde{X}}+\tilde{P}_{, \tilde{X} K}\right) .
\end{aligned}
$$

It is worth noting that even though $P_{, X^{I J} K}$ seems to be a bit complicated, we can show that

$$
P_{, X^{I J} K} \dot{\phi}^{J}=\frac{1}{2} G_{L M, K} \dot{\phi}^{L} \dot{\phi}^{M} \dot{\phi}_{I} \tilde{P}_{, \tilde{X} \tilde{X}}+\dot{\phi}_{I} \tilde{P}_{, \tilde{X} K}+G_{I J, K} \dot{\phi}^{J} \tilde{P}_{, \tilde{X}},
$$

which is just the same form as the K-inflation case. We can also show that

$$
P_{, X^{I J}} \dot{\phi}^{I} \dot{\phi}^{J}=2 X \tilde{P}_{, \tilde{X}} \cdot
$$

The orthonormality conditions for the basis give

$$
e_{n}^{I} e_{m I}=\frac{1}{\tilde{P}_{, \tilde{X}} c_{s}^{2}} \delta_{m n}-\frac{1}{\tilde{P}_{, \tilde{X}}} \frac{1-c_{s}^{2}}{c_{s}^{2}} \delta_{m 1} \delta_{n 1} .
$$


Using these results, the second order action can be written in terms of the decomposed perturbations as

$$
\begin{aligned}
S_{(2)}= & \frac{1}{2} \int d t d^{3} x a^{3}\left[\frac{1}{c_{s}^{2}} \delta_{m n}\left(D_{t} Q_{m}\right)\left(D_{t} Q_{n}\right)-\frac{1}{a^{2}} \delta_{m n} \partial_{i} Q_{m} \partial^{i} Q_{n}\right. \\
& \left.-\mathcal{M}_{m n} Q_{m} Q_{n}+\mathcal{N}_{m n} Q_{n}\left(D_{t} Q_{m}\right)\right] .
\end{aligned}
$$

Unlike the K-inflation models, all field perturbations have the same sound speeds as was pointed out by Ref. [31]. In order to understand the difference between K-inflation and the DBI-inflation, we will consider a generalized model where both cases are included.

\section{Generalized case}

Let us consider models described by

$$
P\left(X^{I J}, \phi^{I}\right)=\tilde{P}\left(Y, \phi^{I}\right)
$$

where

$$
Y=G_{I J}\left(\phi^{K}\right) X^{I J}+\frac{b\left(\phi^{K}\right)}{2}\left(X^{2}-X_{I}^{J} X_{J}^{I}\right) .
$$

The functional form of $Y$ is chosen so that $Y=X \equiv G_{I J} X^{I J}$ in the background as in the DBI-inflation model. This model includes as particular cases the K-inflation model for $b=0$ and the DBI-inflation for $b=-2 f$ and if $\tilde{P}$ has the DBI form. This might be surprising as the DBI action contains additional terms of order $f^{2}$ and $f^{3}$ in $\tilde{X}$ (see equations (49) and (50)), but it turns out that these terms do not contribute to the second order action and the leading order third order action.

Following a similar procedure to the previous subsection, the second order action can be written in terms of the decomposed perturbations as

$$
\begin{aligned}
S_{(2)}= & \frac{1}{2} \int d t d^{3} x a^{3}\left[\left\{\delta_{m n}+\frac{2 X \tilde{P}_{, Y Y}}{\tilde{P}_{, Y}} \delta_{1 m} \delta_{1 n}+\frac{b X}{1+b X}\left(\delta_{n 1} \delta_{m 1}-\delta_{m n}\right)\right\}\left(D_{t} Q_{m}\right)\left(D_{t} Q_{n}\right)\right. \\
& \left.-\frac{1}{a^{2}} \delta_{m n} \partial_{i} Q_{m} \partial^{i} Q_{n}-\mathcal{M}_{m n} Q_{m} Q_{n}+\mathcal{N}_{m n} Q_{n}\left(D_{t} Q_{m}\right)\right]
\end{aligned}
$$

Now we are in a position to explain the difference between K-inflation and DBI-inflation. As in the K-inflation case, the non-trivial second derivative of $P$ affects only the adiabatic perturbations. On the other hand, the non-linear terms of $X^{I J}$ in $Y$ only affects the entropy perturbations as they vanish in the background. Then the sound speed for adiabatic perturbations $c_{a d}^{2}$ and for entropy perturbations $c_{e n}^{2}$ are given by

$$
c_{a d}^{2} \equiv \frac{\tilde{P}_{, Y}}{\tilde{P}_{, Y}+2 X \tilde{P}_{, Y Y}}, \quad c_{e n}^{2} \equiv 1+b X,
$$

and they are independently determined by $\tilde{P}_{, Y Y}$ and $d^{2} Y /\left(d X^{I J} d X^{K L}\right)$ respectively. Thus in general they are different. Let us derive the condition under which the two sound speeds are the same, i.e., $c_{a d}^{2}=c_{e n}^{2}$. This condition is given by

$$
2 X \frac{\tilde{P}_{, Y Y}}{\tilde{P}_{, Y}}=-\frac{b X}{1+b X} .
$$

Then we find that the DBI action is a solution of this equation where $b=-2 f[39]$.

\section{THE LEADING ORDER IN SLOW-ROLL THREE POINT FUNCTION}

In this section, we will calculate the leading order in slow-roll third order action for the generalized model of the previous subsection and then we shall calculate the leading order three point function for both adiabatic and entropy directions. Finally we will obtain the three point function of the comoving curvature perturbation. 


\section{A. Approximations: slow-roll}

In order to control the calculations and to obtain analytical results we need to make use of some approximations. We will use the slow-roll approximation, where we define a set of parameters and assume that these parameters are always small until the end of inflation. We define the slow-roll parameters as

$$
\begin{gathered}
\epsilon \equiv-\frac{\dot{H}}{H^{2}}=\frac{X \tilde{P}_{, Y}}{H^{2}}, \quad \eta \equiv \frac{\dot{\epsilon}}{\epsilon H} \\
\chi_{a d} \equiv \frac{\dot{c}_{a d}}{c_{a d} H}, \quad \chi_{e n} \equiv \frac{\dot{c}_{e n}}{c_{e n} H} .
\end{gathered}
$$

It is important to note that these slow-roll parameters are more general than the usual slow-roll parameters and that their smallness does not necessarily imply that the fields are rolling slowly. Assuming that the parameters $\chi_{a d}$ and $\chi_{e n}$ are small implies that the rates of change of the adiabatic and entropy sound speeds are small, but the sound speeds themselves can have any value between zero and one.

It is convenient to define a parameter that describes the non-linear dependence of the lagrangian on the kinetic term as

$$
\lambda \equiv \frac{2}{3} X^{3} \tilde{P}_{, Y Y Y}+X^{2} \tilde{P}_{, Y Y}
$$

We will also assume that the rate of change of this new parameter is small, as given by

$$
l \equiv \frac{\dot{\lambda}}{\lambda H}
$$

At the end of this section, we will show that the size of the leading order three point function of the fields is fully determined by five parameters evaluated at horizon crossing: $\epsilon, \lambda, H$ and both sound speeds.

It turns out that the equations of motion for both adiabatic and entropy perturbations at first order form a coupled system of second order linear differential equations, see Appendix for details. In general, the coupling (denoted by $\xi$ in equation (A10) ) between adiabatic and entropy modes cannot be neglected but in this work we will study the simpler decoupled case, where we assume that $\xi$ is small when the scales of interest cross outside the sound horizons, i.e., we will assume that $\xi \sim \mathcal{O}(\epsilon)$. With these approximations the adiabatic and entropy modes are decoupled and the system of equations of motion can be solved analytically. For simplicity, we will also assume that the mass term present in the entropy equation of motion is small, i.e., $\mu_{s}^{2} / H^{2} \ll 1$ (refer to Appendix for more details). When calculating the leading order three point functions, we assume that the quantities related to the time derivatives of the basis vectors given by $Z_{m n}$ are also slow-roll suppressed. Finally, the calculation of the three point functions in the next subsections is valid in the limit of small sound speeds. Our results will also include sub-leading terms of $\mathcal{O}(1)$ but these terms will in general (for small sound speeds) receive corrections coming from terms of the order of $\epsilon / c_{s}^{2}$, that we have neglected.

\section{B. Third order action at leading order}

At leading order in the previous approximations, the third order action for the general model (1) is calculated as

$$
\begin{aligned}
S_{(3)}= & \frac{1}{2} \int d x^{3} d t a^{3}\left[P_{, X^{I K} X^{J L}} \dot{\phi}^{(I} \dot{Q}^{K)} \dot{Q}^{J} \dot{Q}^{L}+\frac{1}{3} P_{, X^{I K} X^{J L} X^{M N}} \dot{\phi}^{(I} \dot{Q}^{K)} \dot{\phi}^{(J} \dot{Q}^{L)} \dot{\phi}^{(M} \dot{Q}^{N)}\right. \\
& \left.-\frac{1}{a^{2}} P_{, X^{I K} X^{J L}} \dot{\phi}^{(I} \dot{Q}^{K)} \partial_{i} Q^{J} \partial^{i} Q^{L}\right]
\end{aligned}
$$

After decomposition into the new adiabatic/entropy basis the third order action can be written as

$$
S_{(3)}=\int d x^{3} d t a^{3}\left[\frac{1}{2} \Xi_{n m l} \dot{Q}_{n} \dot{Q}_{m} \dot{Q}_{l}-\frac{1}{2 a^{2}} \Upsilon_{n m l} \dot{Q}_{n}\left(\partial_{i} Q_{m}\right)\left(\partial^{i} Q_{l}\right)\right]
$$


where we define the coefficients $\Xi_{n m l}$ and $\Upsilon_{n m l}$ as

$$
\begin{aligned}
\Xi_{n m l}= & P_{, X^{I K} X^{J L}} \sqrt{P_{, X^{M N}} \dot{\phi}^{M} \dot{\phi}^{N}} e_{1}^{(I} e_{(n}^{K)} e_{m}^{J} e_{l)}^{L} \\
& +\frac{1}{3} P_{, X^{I K} X^{J L} X^{M N}}\left(P_{, X^{P Q}} \dot{\phi}^{P} \dot{\phi}^{Q}\right)^{3 / 2} e_{1}^{(I} e_{n}^{K)} e_{1}^{(J} e_{m}^{L)} e_{1}^{(M} e_{l}^{N)}, \\
\Upsilon_{n m l}= & P_{X^{I K} X^{J L}} \sqrt{P_{, X^{M N}} \dot{\phi}^{M} \dot{\phi}^{N}} e_{1}^{(I} e_{n}^{K)} e_{m}^{J} e_{l}^{L} .
\end{aligned}
$$

We shall now give some useful formulae of the previous quantities for the different inflationary models considered in this work.

\section{K-inflation}

For the K-inflation model we have

$$
\begin{gathered}
\Xi_{n m l}=\left(2 X \tilde{P}_{, X}\right)^{-\frac{1}{2}}\left(\frac{2 X \tilde{P}_{, X X}}{\tilde{P}_{, X}} \delta_{1(n} \delta_{m l)}+\frac{4}{3} \frac{X^{2} \tilde{P}_{, X X X}}{\tilde{P}_{, X}} \delta_{n 1} \delta_{m 1} \delta_{l 1}\right), \\
\Upsilon_{n m l}=\left(2 X \tilde{P}_{, X}\right)^{-\frac{1}{2}} \frac{2 X \tilde{P}_{, X X}}{\tilde{P}_{, X}} \delta_{n 1} \delta_{m l} . \\
\text { 2. DBI-inflation }
\end{gathered}
$$

For the DBI-inflation scenario they are given by

$$
\begin{aligned}
& \Xi_{n m l}=\left(2 X \tilde{P}_{, \tilde{X}}\right)^{-\frac{1}{2}} \frac{1-c_{s}^{2}}{c_{s}^{4}} \delta_{1(n} \delta_{m l)}, \\
& \Upsilon_{n m l}=\left(2 X \tilde{P}_{, \tilde{X}}\right)^{-\frac{1}{2}}\left(\frac{1-c_{s}^{2}}{c_{s}^{2}} \delta_{n 1} \delta_{m l}-2 \frac{1-c_{s}^{2}}{c_{s}^{2}}\left(\delta_{n 1} \delta_{m l}-\delta_{n(m} \delta_{l) 1}\right)\right),
\end{aligned}
$$

where $c_{s}^{2}$ should be understood as the sound speed defined in Eq. (52).

\section{Generalized case}

For the generalized case of subsection IVC, $\Xi_{n m l}$ and $\Upsilon_{n m l}$ can be written as

$$
\begin{aligned}
& \Xi_{n m l}=\left(2 X \tilde{P}_{, Y}\right)^{-\frac{1}{2}}\left[\frac{\left(1-c_{a d}^{2}\right)}{c_{a d}^{2} c_{e n}^{2}} \delta_{1(n} \delta_{m l)}+\left(\frac{4}{3} \frac{X^{2} \tilde{P}_{, Y Y Y}}{\tilde{P}_{, Y}}-\frac{\left(1-c_{a d}^{2}\right)\left(1-c_{e n}^{2}\right)}{c_{a d}^{2} c_{e n}^{2}}\right) \delta_{n 1} \delta_{m 1} \delta_{l 1}\right], \\
& \Upsilon_{n m l}=\left(2 X \tilde{P}_{, Y}\right)^{-\frac{1}{2}}\left(\frac{1-c_{a d}^{2}}{c_{a d}^{2}} \delta_{n 1} \delta_{m l}-\frac{2\left(1-c_{e n}^{2}\right)}{c_{e n}^{2}}\left(\delta_{n 1} \delta_{m l}-\delta_{n(m} \delta_{l) 1}\right)\right)
\end{aligned}
$$

and it is obvious that the DBI-inflation is a specific case of the general model with $c_{a d}^{2}=c_{e n}^{2}=c_{s}^{2}$.

\section{The three point functions of the fields}

In this subsection, we derive the three point functions of the adiabatic and entropy fields in the generalized case and at leading order in slow-roll and in the small sound speeds limit. We consider the two-field case with the adiabatic field $\sigma$ and the entropy field $s$.

The perturbations are promoted to quantum operators like

$$
Q_{n}(\tau, \mathbf{x})=\frac{1}{(2 \pi)^{3}} \int d^{3} \mathbf{k} Q_{n}(\tau, \mathbf{k}) e^{i \mathbf{k} \cdot \mathbf{x}},
$$


where

$$
Q_{n}(\tau, \mathbf{k})=u_{n}(\tau, \mathbf{k}) a_{n}(\mathbf{k})+u_{n}^{*}(\tau,-\mathbf{k}) a_{n}^{\dagger}(-\mathbf{k}) .
$$

$a_{n}(\mathbf{k})$ and $a_{n}^{\dagger}(-\mathbf{k})$ are the annihilation and creation operator respectively, that satisfy the usual commutation relations

$$
\left[a_{n}\left(\mathbf{k}_{\mathbf{1}}\right), a_{m}^{\dagger}\left(\mathbf{k}_{\mathbf{2}}\right)\right]=(2 \pi)^{3} \delta^{(3)}\left(\mathbf{k}_{\mathbf{1}}-\mathbf{k}_{\mathbf{2}}\right) \delta_{n m}, \quad\left[a_{n}\left(\mathbf{k}_{\mathbf{1}}\right), a_{m}\left(\mathbf{k}_{\mathbf{2}}\right)\right]=\left[a_{n}^{\dagger}\left(\mathbf{k}_{\mathbf{1}}\right), a_{m}^{\dagger}\left(\mathbf{k}_{\mathbf{2}}\right)\right]=0 .
$$

At leading order the solution for the mode functions is (see Appendix for details)

$$
u_{n}(\tau, \mathbf{k})=A_{n} \frac{1}{k^{3 / 2}}\left(1+i k c_{n} \tau\right) e^{-i k c_{n} \tau},
$$

where $c_{n}$ stands for either the adiabatic or the entropy sound speeds.

The two point correlation function is

$$
\left\langle 0\left|Q_{n}\left(\tau=0, \mathbf{k}_{\mathbf{1}}\right) Q_{m}\left(\tau=0, \mathbf{k}_{\mathbf{2}}\right)\right| 0\right\rangle=(2 \pi)^{3} \delta^{(3)}\left(\mathbf{k}_{\mathbf{1}}+\mathbf{k}_{\mathbf{2}}\right) \mathcal{P}_{Q_{n}} \frac{2 \pi^{2}}{k_{1}^{3}} \delta_{n m},
$$

where the power spectrum is defined as

$$
\mathcal{P}_{Q_{n}}=\frac{\left|A_{n}\right|^{2}}{2 \pi^{2}}, \quad\left|A_{\sigma}\right|^{2}=\frac{H^{2}}{2 c_{a d}}, \quad\left|A_{s}\right|^{2}=\frac{H^{2}}{2 c_{e n}},
$$

and it should be evaluated at the time of horizon crossing $c_{n *} k_{1}=a_{*} H_{*}$

The vacuum expectation value of the three point operator in the interaction picture (at first order) is [34, 40]

$$
\left\langle\Omega\left|Q_{l}\left(t, \mathbf{k}_{\mathbf{1}}\right) Q_{m}\left(t, \mathbf{k}_{\mathbf{2}}\right) Q_{n}\left(t, \mathbf{k}_{\mathbf{3}}\right)\right| \Omega\right\rangle=-i \int_{t_{0}}^{t} d \tilde{t}\left\langle 0\left|\left[Q_{l}\left(t, \mathbf{k}_{\mathbf{1}}\right) Q_{m}\left(t, \mathbf{k}_{\mathbf{2}}\right) Q_{n}\left(t, \mathbf{k}_{\mathbf{3}}\right), H_{I}(\tilde{t})\right]\right| 0\right\rangle,
$$

where $t_{0}$ is some early time during inflation when the field's vacuum fluctuation are deep inside the horizons, $t$ is some time after horizon exit. $|\Omega\rangle$ is the interacting vacuum which is different from the free theory vacuum $|0\rangle$. If one uses conformal time, it's a good approximation to perform the integration from $-\infty$ to 0 because $\tau \approx-(a H)^{-1}$. $H_{I}$ denotes the interaction hamiltonian and it is given by $H_{I}=-L_{3}$, where $L_{3}$ is the lagrangian obtained from the action (74).

At this order, the only non-zero three point functions are

$$
\begin{aligned}
\left\langle\Omega\left|Q_{\sigma}\left(0, \mathbf{k}_{\mathbf{1}}\right) Q_{\sigma}\left(0, \mathbf{k}_{\mathbf{2}}\right) Q_{\sigma}\left(0, \mathbf{k}_{\mathbf{3}}\right)\right| \Omega\right\rangle= & (2 \pi)^{3} \delta^{(3)}\left(\mathbf{k}_{\mathbf{1}}+\mathbf{k}_{\mathbf{2}}+\mathbf{k}_{\mathbf{3}}\right) \frac{2 c_{a d}\left|A_{\sigma}\right|^{6}}{H} \frac{1}{\Pi_{i=1}^{3} k_{i}^{3}} \frac{1}{K} \\
& \times\left[6 c_{a d}^{2}\left(C_{3}+C_{4}\right) \frac{k_{1}^{2} k_{2}^{2} k_{3}^{2}}{K^{2}}-C_{1} k_{1}^{2} \mathbf{k}_{\mathbf{2}} \cdot \mathbf{k}_{\mathbf{3}}\left(1+\frac{k_{2}+k_{3}}{K}+2 \frac{k_{2} k_{3}}{K^{2}}\right)\right. \\
& +2 \text { cyclic terms }],
\end{aligned}
$$

$$
\begin{aligned}
\left\langle\Omega\left|Q_{\sigma}\left(0, \mathbf{k}_{\mathbf{1}}\right) Q_{s}\left(0, \mathbf{k}_{\mathbf{2}}\right) Q_{s}\left(0, \mathbf{k}_{\mathbf{3}}\right)\right| \Omega\right\rangle= & (2 \pi)^{3} \delta^{(3)}\left(\mathbf{k}_{\mathbf{1}}+\mathbf{k}_{\mathbf{2}}+\mathbf{k}_{\mathbf{3}}\right) \frac{\left|A_{\sigma}\right|^{2}\left|A_{s}\right|^{4}}{H} \frac{1}{\Pi_{i=1}^{3} k_{i}^{3}} \frac{1}{\tilde{K}} \\
& \times\left[C_{2} c_{e n}^{2} k_{3}^{2} \mathbf{k}_{\mathbf{1}} \cdot \mathbf{k}_{\mathbf{2}}\left(1+\frac{c_{a d} k_{1}+c_{e n} k_{2}}{\tilde{K}}+\frac{2 c_{a d} c_{e n} k_{1} k_{2}}{\tilde{K}^{2}}\right)+\left(k_{2} \leftrightarrow k_{3}\right)\right. \\
& \left.+4 C_{3} c_{a d}^{2} c_{e n}^{4} \frac{k_{1}^{2} k_{2}^{2} k_{3}^{2}}{\tilde{K}^{2}}-2\left(C_{1}+C_{2}\right) c_{a d}^{2} k_{1}^{2} \mathbf{k}_{\mathbf{2}} \cdot \mathbf{k}_{\mathbf{3}}\left(1+c_{e n} \frac{k_{2}+k_{3}}{\tilde{K}}+2 c_{e n}^{2} \frac{k_{2} k_{3}}{\tilde{K}^{2}}\right)\right],
\end{aligned}
$$

where $K=k_{1}+k_{2}+k_{3}, \tilde{K}=c_{a d} k_{1}+c_{e n}\left(k_{2}+k_{3}\right)$, cyclic terms means cyclic permutations of the three wave vectors and $\left(k_{2} \leftrightarrow k_{3}\right)$ denotes a term like the preceding one but with $k_{2}$ and $k_{3}$ interchanged. The pure adiabatic three point function is evaluated at the moment $\tau_{*}$ at which the total wave number $K$ exits the horizon, i.e., when $K c_{a d *}=a_{*} H_{*}$. Because of the different propagation speeds, the adiabatic and entropy modes become classical at different times, however at leading order we assume that the background dependent coefficients of (91) do not vary with time and so they can also be evaluated at the moment $\tau_{*}$. 
The different constants $C_{N}$ are given by

$$
\begin{aligned}
& C_{1}=\left(2 H^{2} \epsilon\right)^{-\frac{1}{2}} \frac{1-c_{a d}^{2}}{c_{a d}^{2}}, \quad C_{2}=-2\left(2 H^{2} \epsilon\right)^{-\frac{1}{2}} \frac{1-c_{e n}^{2}}{c_{e n}^{2}}, \\
& C_{3}=\left(2 H^{2} \epsilon\right)^{-\frac{1}{2}} \frac{1-c_{a d}^{2}}{c_{a d}^{2} c_{e n}^{2}}, \quad C_{4}=\left(2 H^{2} \epsilon\right)^{-\frac{1}{2}}\left(\frac{2 \lambda}{H^{2} \epsilon}-\frac{1-c_{a d}^{2}}{c_{a d}^{2} c_{e n}^{2}}\right) .
\end{aligned}
$$

\section{The three point function of the comoving curvature perturbation}

In this subsection, we calculate the leading order in slow-roll three point function of the comoving curvature perturbation in terms of three point function of the fields obtained in the previous subsection.

During the inflationary era the comoving curvature perturbation is given by

$$
\mathcal{R}=\frac{H}{\dot{\sigma}} \frac{Q_{\sigma}}{\sqrt{\tilde{P}_{, Y}}} .
$$

It is convenient to define the entropy perturbation $\mathcal{S}$ as

$$
S=\frac{H}{\dot{\sigma}} \frac{Q_{s}}{\sqrt{\tilde{P}_{, Y}}} \sqrt{\frac{c_{e n}}{c_{a d}}}
$$

so that $\mathcal{P}_{\mathcal{S}_{*}} \simeq \mathcal{P}_{\mathcal{R}_{*}}$, where the subscript $*$ means that the quantity should be evaluated at horizon crossing.

In this work we will ignore the possibility that the entropy perturbations during inflation can lead to primordial entropy perturbations that could be observable in the CMB. But we shall consider the effect of entropy perturbations on the final curvature perturbation. We will follow the analysis of Wands et.al. [41], where it has been shown that even on large scales the curvature perturbation can change in time because of the presence of entropy perturbations. The way the entropy perturbations are converted to curvature perturbations is model dependent but it was shown that this model dependence can be parameterized by a transfer coefficient $T_{\mathcal{R S}}$ [41] like

$$
\mathcal{R}=\mathcal{R}_{*}+T_{\mathcal{R S}} \mathcal{S}_{*}=\mathcal{A}_{\sigma} Q_{\sigma *}+\mathcal{A}_{s} Q_{s *},
$$

with

$$
\mathcal{A}_{\sigma}=\left(\frac{H}{\dot{\sigma} \sqrt{\tilde{P}_{, Y}}}\right)_{*}, \quad \mathcal{A}_{s}=T_{\mathcal{R S}}\left(\frac{H}{\dot{\sigma} \sqrt{\tilde{P}_{, Y}}} \sqrt{\frac{c_{e n}}{c_{a d}}}\right)_{*} .
$$

Using the previous expressions we can now relate the three point function of the curvature perturbation to the three point functions of the fields obtained in the previous subsection. The three point function of the curvature perturbation is given by

$$
\left\langle\mathcal{R}\left(\mathbf{k}_{\mathbf{1}}\right) \mathcal{R}\left(\mathbf{k}_{\mathbf{2}}\right) \mathcal{R}\left(\mathbf{k}_{\mathbf{3}}\right)\right\rangle=\mathcal{A}_{\sigma}^{3}\left\langle Q_{\sigma}\left(\mathbf{k}_{\mathbf{1}}\right) Q_{\sigma}\left(\mathbf{k}_{\mathbf{2}}\right) Q_{\sigma}\left(\mathbf{k}_{\mathbf{3}}\right)\right\rangle+\mathcal{A}_{\sigma} \mathcal{A}_{s}^{2}\left(\left\langle Q_{\sigma}\left(\mathbf{k}_{\mathbf{1}}\right) Q_{s}\left(\mathbf{k}_{\mathbf{2}}\right) Q_{s}\left(\mathbf{k}_{\mathbf{3}}\right)\right\rangle+2 \text { perms. }\right) .
$$

For the DBI-inflation case the previous equation can be simplified and the total momentum dependence of the three point function of the comoving curvature perturbation is the same as in single field DBI [31]. For our general model this is no longer the case, i.e., the different terms of the previous equation have different momentum dependence. Once again one can see that DBI-inflation is a very particular case and more importantly it provides a distinct signature that enables us to distinguish it from other more general models.

\section{CONCLUSION}

In this paper, we studied the non-gaussianity from the bispectrum in general multi-field inflation models with a generic kinetic term. Our model is fairly generic including the K-inflation and the DBI-inflation as special cases. We derived the second and third order actions for the perturbations including the effect of gravity. The second order action is written in terms of adiabatic and entropy perturbations. It was shown that the sound speeds for these perturbations are in general different. In the K-inflation the entropy perturbations propagate at the speed of light. 
The DBI-inflation is a special case where the sound speed for the entropy perturbations is the same as the adiabatic sound speed. We found that, from the requirement that the sound speeds for adiabatic and entropy perturbations are the same, we obtain the DBI form for the action.

Then we derive the three point function in the small sound speeds limit at leading order in slow-roll expansion. In these approximations there exists a three point function between adiabatic perturbations $Q_{\sigma}$ and entropy perturbations $Q_{s},\left\langle Q_{\sigma}\left(\mathbf{k}_{\mathbf{1}}\right) Q_{s}\left(\mathbf{k}_{\mathbf{2}}\right) Q_{s}\left(\mathbf{k}_{\mathbf{3}}\right)\right\rangle$, in addition to the pure adiabatic three point function. This mixed contribution has a different momentum dependence if the sound speed for the entropy perturbations is different from the adiabatic one. This provides a possibility to distinguish between the multi-field models and the single field models. Unfortunately, in the multi-field DBI case, the sound speed for the entropy perturbation is the same as the adiabatic one and the mixed contribution only changes the amplitude of the three point function. This could help to ease the constraints on DBI-inflation as is discussed in Ref. [31].

In order to calculate the effect of the entropy perturbations on the curvature perturbation at the recombination, we need to specify a model that describes how the entropy perturbations are converted to the curvature perturbations. In addition, even during inflation, if the trajectory in field space changes non-trivially, the entropy perturbations can be converted to the curvature perturbation. In this paper, we assumed that this does not happen and neglected a mixing between the curvature and entropy perturbations. It would be interesting to study this mixing in specific string theory motivated models.

Note added: While we were writing up this work, similar results appeared on the arXiv [44].

\section{Acknowledgments}

We would like to thank G. Tasinato for useful discussions. FA is supported by "Fundação para a Ciência e Tecnologia (Portugal)", with the fellowship's reference number: SFRH/BD/18116/2004. SM is supported by a JSPS Research Fellowship and JSPS Core-to-Core Program "International Research Network for Dark Energy". SM is grateful to the ICG, Portsmouth, for their hospitality when this work was initiated. KK is supported by STFC.

\section{APPENDIX A: EQUATIONS OF MOTION FOR THE FLUCTUATIONS}

Here, we derive the equations of motion for linear perturbations for the generalized model introduced in IV C In terms of the field space "covariant quantities" [42] which are given by

$$
\begin{aligned}
& \mathcal{D}_{t} \dot{\phi}^{I} \equiv \ddot{\phi}^{I}+\Gamma_{J K}^{I} \dot{\phi}^{J} \dot{\phi}^{K}, \\
& \mathcal{D}_{t} Q^{I} \equiv \dot{Q}^{I}+\Gamma_{J K}^{I} \dot{\phi}^{J} Q^{K}, \\
& \mathcal{D}_{I} \mathcal{D}_{J} \tilde{P} \equiv \tilde{P}_{, I J}-\Gamma_{I J}^{K} \tilde{P}_{, K}, \\
& \mathcal{R}_{K L J}^{I} \equiv \Gamma_{K J, L}^{I}-\Gamma_{K L, J}^{I}+\Gamma_{L M}^{I} \Gamma_{J K}^{M}-\Gamma_{J M}^{I} \Gamma_{L K}^{M},
\end{aligned}
$$

$\left(\Gamma_{J K}^{I}\right.$ denotes the Christoffel symbols associated with the field space metric $\left.G_{I J}\right)$, the second order action can be expressed as

$$
\begin{aligned}
S_{(2)}= & \frac{1}{2} \int d t d^{3} x a^{3}\left[\left(\tilde{P}_{, Y} G_{I J}+\tilde{P}_{, Y Y} \dot{\phi}_{I} \dot{\phi}_{J}\right) \mathcal{D}_{t} Q^{I} \mathcal{D}_{t} Q^{J}\right. \\
& \left.-\frac{1}{a^{2}} \tilde{P}_{, Y}\left[(1+b X) G_{I J}-b X_{I J}\right] \partial_{i} Q^{I} \partial^{i} Q^{J}-\overline{\mathcal{M}}_{I J} Q^{I} Q^{J}+2 \tilde{P}_{, Y J} \dot{\phi}_{I} Q^{J} \mathcal{D}_{t} Q^{I}\right],
\end{aligned}
$$

with the effective squared mass matrix

$$
\begin{aligned}
\overline{\mathcal{M}}_{I J}= & -\mathcal{D}_{I} \mathcal{D}_{J} \tilde{P}-\tilde{P}_{, Y} \mathcal{R}_{I K L J} \dot{\phi}^{K} \dot{\phi}^{L}+\frac{X \tilde{P}_{Y Y}}{H}\left(\tilde{P}_{, Y J} \dot{\phi}_{I}+\tilde{P}_{, Y I} \dot{\phi}_{J}\right) \\
& +\frac{X \tilde{P}_{Y Y}^{3}}{2 H^{2}}\left(1-\frac{1}{c_{a d}^{2}}\right) \dot{\phi}_{I} \dot{\phi}_{J}-\frac{1}{a^{3}} \mathcal{D}_{t}\left[\frac{a^{3}}{2 H} \tilde{P}_{, Y}^{2}\left(1+\frac{1}{c_{a d}^{2}}\right) \dot{\phi}_{I} \dot{\phi}_{J}\right] .
\end{aligned}
$$

It is worth noting that except for the coefficients of the kinetic term and the gradient term, this action is the same as the K-inflation case and DBI-inflation case which are derived in [29] and [31], respectively. 
From now on we will derive the equations of motion for the fluctuations. For simplicity, let us now restrict our attention to the two field case $(I=1,2)$. Then, the perturbations can be decomposed into $Q^{I}=Q_{\sigma} e_{\sigma}^{I}+Q_{s} e_{s}^{I}$, where $e_{\sigma}^{I}=e_{1}^{I}$ and $e_{s}^{I}$ is the unit vector orthogonal to $e_{\sigma}^{I}$. As in standard inflation, it is more convenient to use conformal time $\tau=\int d t / a(t)$ and define the canonically normalized fields [43]

$$
v_{\sigma} \equiv \frac{a}{c_{a d}} Q_{\sigma}, \quad v_{s} \equiv \frac{a}{c_{e n}} Q_{s}
$$

From the similar calculations with K-inflation and DBI-inflation cases analyzed by [29] and [31], respectively, we find the equations of motion for $v_{\sigma}$ and $v_{s}$ as

$$
\begin{aligned}
v_{\sigma}^{\prime \prime}-\xi v_{s}^{\prime}+\left(c_{a d}^{2} k^{2}-\frac{z^{\prime \prime}}{z}\right) v_{\sigma}-\frac{(z \xi)^{\prime}}{z} v_{s} & =0, \\
v_{s}^{\prime \prime}+\xi v_{\sigma}^{\prime}+\left(c_{e n}^{2} k^{2}-\frac{\alpha^{\prime \prime}}{\alpha}+a^{2} \mu_{s}^{2}\right) v_{s}-\frac{z^{\prime}}{z} \xi v_{\sigma} & =0,
\end{aligned}
$$

where the primes denote the derivative with respect to $\tau$ and

$$
\begin{aligned}
\xi & \equiv \frac{a}{\dot{\sigma} \tilde{P}_{, Y} c_{a d}}\left[\left(1+c_{a d}^{2}\right) \tilde{P}_{, s}-c_{a d}^{2} \dot{\sigma}^{2} \tilde{P}_{, Y s}\right] \\
\mu_{s}^{2} & \equiv-\frac{\tilde{P}_{, s s}}{\tilde{P}_{, Y}}+\frac{1}{2} \dot{\sigma}^{2} \tilde{R}-\frac{1}{2 c_{a d}^{2} X} \frac{\tilde{P}_{, s}^{2}}{\tilde{P}_{, Y}^{2}}+2 \frac{\tilde{P}_{, Y s} \tilde{P}_{, s}}{\tilde{P}_{, Y}^{2}} \\
z & \equiv \frac{a \dot{\sigma}}{c_{a d} H} \sqrt{\tilde{P}_{, Y}}, \quad \alpha \equiv a \sqrt{\tilde{P}_{, Y}}
\end{aligned}
$$

with

$$
\dot{\sigma} \equiv \sqrt{2 X}, \quad \tilde{P}_{, s} \equiv \tilde{P}_{, I} e_{s}^{I} \sqrt{\tilde{P}_{, Y}} c_{e n}, \quad \tilde{P}_{, Y s} \equiv \tilde{P}_{, Y I} e_{s}^{I} \sqrt{\tilde{P}_{, Y}} c_{e n}, \quad \tilde{P}_{, s s} \equiv\left(\mathcal{D}_{I} \mathcal{D}_{J} \tilde{P}\right) e_{s}^{I} e_{s}^{J} \tilde{P}_{, Y} c_{e n}^{2}
$$

and $\tilde{R}$ denotes the Riemann scalar curvature of the field space.

If we assume that the effect of the coupling $\xi$ can be neglected when the scales of interest cross the sound horizons the two degrees of freedom are decoupled and the system can be easily quantized. If we further assume the slow-roll approximations, the time evolution of $H, c_{a d}$, and $\dot{\sigma}$ is small with respect to that of the scale factor and the relations $z^{\prime \prime} / z \simeq 2 / \tau^{2}$ and $\alpha^{\prime \prime} / \alpha \simeq 2 / \tau^{2}$ hold (see section V.A for these approximations). The solutions of (A8) and (A9) with the Bunch-Davies vacuum initial conditions are thus given by

$$
\begin{aligned}
& v_{\sigma k} \simeq \frac{1}{\sqrt{2 k c_{a d}}} e^{-i k c_{a d} \tau}\left(1-\frac{i}{k c_{a d} \tau}\right) \\
& v_{s k} \simeq \frac{1}{\sqrt{2 k c_{e n}}} e^{-i k c_{e n} \tau}\left(1-\frac{i}{k c_{e n} \tau}\right)
\end{aligned}
$$

when $\mu_{s}^{2} / H^{2}$ is negligible for the entropy mode.

Therefore, the power spectra for $Q_{\sigma}$ and $Q_{s}$ are obtained as

$$
\mathcal{P}_{Q_{\sigma}} \simeq \frac{H^{2}}{4 \pi^{2} c_{a d}}, \quad \mathcal{P}_{Q_{s}} \simeq \frac{H^{2}}{4 \pi^{2} c_{e n}},
$$

which are evaluated at sound horizon crossing. The ratio of the power spectra for the adiabatic and entropy modes is thus given by $\mathcal{P}_{Q_{s}} / \mathcal{P}_{Q_{\sigma}}=c_{a d} / c_{e n}$.

[1] N. Bartolo, E. Komatsu, S. Matarrese and A. Riotto, Phys. Rept. 402, 103 (2004) arXiv:astro-ph/0406398.

[2] http://www.rssd.esa.int/index.php?project=Planck

[3] C. Armendariz-Picon, T. Damour and V. F. Mukhanov, Phys. Lett. B 458 (1999) 209 arXiv:hep-th/9904075.

[4] J. Garriga and V. F. Mukhanov, Phys. Lett. B 458 (1999) 219 arXiv:hep-th/9904176.

[5] E. Silverstein and D. Tong, Phys. Rev. D 70 (2004) 103505 arXiv:hep-th/0310221.

[6] M. Alishahiha, E. Silverstein and D. Tong, Phys. Rev. D 70 (2004) 123505 arXiv:hep-th/0404084. 
[7] X. Chen, Phys. Rev. D 71 (2005) 063506 arXiv:hep-th/0408084.

[8] X. Chen, JHEP 0508 (2005) 045 arXiv:hep-th/0501184.

[9] X. Chen, Phys. Rev. D 72 (2005) 123518 arXiv:astro-ph/0507053.

[10] X. Chen, M. x. Huang, S. Kachru and G. Shiu, JCAP 0701 (2007) 002 arXiv:hep-th/0605045].

[11] M. x. Huang and G. Shiu, Phys. Rev. D 74 (2006) 121301 arXiv:hep-th/0610235.

[12] F. Arroja and K. Koyama, Phys. Rev. D 77, 083517 (2008) [arXiv:0802.1167 [hep-th]].

[13] S. Kecskemeti, J. Maiden, G. Shiu and B. Underwood, JHEP 0609 (2006) 076 arXiv:hep-th/0605189.

[14] J. E. Lidsey and D. Seery, Phys. Rev. D 75 (2007) 043505 arXiv:astro-ph/0610398.

[15] D. Baumann and L. McAllister, Phys. Rev. D 75 (2007) 123508 arXiv:hep-th/0610285].

[16] R. Bean, S. E. Shandera, S. H. Henry Tye and J. Xu, JCAP 0705 (2007) 004 arXiv:hep-th/0702107.

[17] J. E. Lidsey and I. Huston, JCAP 0707 (2007) 002 arXiv:0705.0240 [hep-th]].

[18] H. V. Peiris, D. Baumann, B. Friedman and A. Cooray, Phys. Rev. D 76 (2007) 103517 arXiv:0706.1240 [astro-ph]].

[19] T. Kobayashi, S. Mukohyama and S. Kinoshita, JCAP 0801 (2008) 028 [arXiv:0708.4285 [hep-th]].

[20] L. Lorenz, J. Martin and C. Ringeval, JCAP 0804 (2008) 001 arXiv:0709.3758 [hep-th]].

[21] A. A. Starobinsky and J. Yokoyama, arXiv:gr-qc/9502002.

[22] G. I. Rigopoulos, E. P. S. Shellard and B. J. W. van Tent, Phys. Rev. D 73 (2006) 083522 arXiv:astro-ph/0506704.

[23] F. Vernizzi and D. Wands, JCAP 0605, 019 (2006) arXiv:astro-ph/0603799].

[24] M. Sasaki, arXiv:0805.0974 [astro-ph].

[25] D. A. Easson, R. Gregory, D. F. Mota, G. Tasinato and I. Zavala, JCAP 0802 (2008) 010 [arXiv:0709.2666 [hep-th]].

[26] Z. Lalak, D. Langlois, S. Pokorski and K. Turzynski, JCAP 0707 (2007) 014 [arXiv:0704.0212 [hep-th]].

[27] R. H. Brandenberger, A. R. Frey and L. C. Lorenz, arXiv:0712.2178 [hep-th].

[28] M. x. Huang, G. Shiu and B. Underwood, Phys. Rev. D 77 (2008) 023511 arXiv:0709.3299 [hep-th]].

[29] D. Langlois and S. Renaux-Petel, JCAP 0804 (2008) 017 arXiv:0801.1085 [hep-th]].

[30] X. Gao, arXiv:0804.1055 [astro-ph].

[31] D. Langlois, S. Renaux-Petel, D. A. Steer and T. Tanaka, arXiv:0804.3139 [hep-th].

[32] Strictly speaking, we adopt the symmetrised derivative, $P_{, X^{I J}} \equiv \frac{1}{2}\left(\frac{\partial P}{\partial X^{I J}}+\frac{\partial P}{\partial X^{J I}}\right)$.

[33] R. Arnowitt, S. Deser and C. W. Misner, Phys. Rev. 117 (1960) 1595.

[34] J. M. Maldacena, JHEP 0305 (2003) 013 arXiv:astro-ph/0210603.

[35] D. Seery and J. E. Lidsey, JCAP 0506 (2005) 003 arXiv:astro-ph/0503692.

[36] D. Seery and J. E. Lidsey, JCAP 0509 (2005) 011 arXiv:astro-ph/0506056.

[37] C. Gordon, D. Wands, B. A. Bassett and R. Maartens, Phys. Rev. D 63 (2001) 023506 arXiv:astro-ph/0009131.

[38] It is worth noting that the normalisation given in [29, 31, 44] is $G_{I J} e_{n}^{I} e_{m}^{J}=\delta_{n m}$, unless $P_{X^{I J}}=G_{I J}$, that is, the case with the canonical kinetic terms, our results written in terms of $Q_{n}$ are different from the results of [29, 31, [44]. But it can be shown that when written in terms of $v_{n}$ which are the cannonical variables for the quantum theory, our results are consistent with the results of [29, 31, 44].

[39] We thank Gianmassimo Tasinato for a discussion on this point. While we were writing up the work, there appeared a paper on the arXiv [44]. It is argued that the speciality of the DBI action comes from the fact that it describes the fluctuations of the positions of the brane in the higher-dimensional spacetime and they propagate at the speed of light. From the point of view of observers on the brane, the sound speeds are smaller than 1 due to the Lorentz factor $1 / c_{s}^{2}$.

[40] S. Weinberg, Phys. Rev. D 72 (2005) 043514 arXiv:hep-th/0506236.

[41] D. Wands, N. Bartolo, S. Matarrese and A. Riotto, Phys. Rev. D 66 (2002) 043520 arXiv:astro-ph/0205253.

[42] M. Sasaki and E. D. Stewart, Prog. Theor. Phys. 95 (1996) 71 arXiv:astro-ph/9507001.

[43] Since the definitions of $Q_{\sigma}$ and $Q_{s}$ in our paper are different from the definitions in [29, 31, 44] the relations between $Q_{\sigma}$ and $v_{\sigma}$, and $Q_{\sigma}$ and $v_{s}$ are also different from the analogous relations in those papers.

[44] D. Langlois, S. Renaux-Petel, D. A. Steer and T. Tanaka, arXiv:0806.0336 [hep-th]. 